\title{
The Pressure Dependence of Self Diffusion in Supercooled Light and Heavy Water
}

\author{
F. X. Prielmeier, E. W. Lang, R. J. Speedy*), and H.-D. Lüdemann**) \\ Institut für Biophysik und Physikalische Biochemie, Universität Regensburg, Postfach 397, D-8400 Regensburg
}

\section{Diffusion / High Pressure / Isotope Effects / Spectroscopy, Nuclear Magnetic Resonance / Transport Properties}

Measurements of self-diffusion coefficients $D$ in $\mathrm{H}_{2} \mathrm{O}$ and $\mathrm{D}_{2} \mathrm{O}$ at pressures up to $400 \mathrm{MPa}$ and temperatures down to $200 \mathrm{~K}$ are reported. Upon cooling molecular motions are strongly retarded leading to a pronounced non-Arrhenius temperature dependence of $D$. In contrast, initial compression of cold water facilitates translational and rotational motions. Unexpectedly, the rates of translational diffusion increase less dramatically than those of rotational diffusion. These results are discussed in relation to the peculiar structure of the hydrogen bond network of cold water. Further all dynamic isotope effects can be removed by shifting all temperature scales by $\sim 7 \mathrm{~K}$ in going from $\mathrm{H}_{2} \mathrm{O}$ to $\mathrm{D}_{2} \mathrm{O}$.

\section{Introduction}

Water exhibits many unusual properties which in their combination make it unique among molecular liquids $[1-4]$. They all originate from long-ranged structural correlations which develop within the random, transient hydrogen-bonded network in liquid water at low temperatures. Their correlation length increases with falling temperature, as deduced from the behaviour of thermodynamic response functions. Speedy and Angell [3,5] first showed that decreasing $T$ below $T_{\mathrm{m}}$ increases density fluctuations. Bosio et al. [7] confirmed that the predicted increase in the densitydensity correlation length is observable by small angle $\mathrm{X}$-ray scattering. The behaviour of transport and relaxation coefficients further also implies a strong slowing down of these structural fluctuations upon cooling. But application of hydrostatic pressure reduces the correlation length and enhances molecular mobility. Obviously compression of the liquid must modify these fluctuations and it might be expected, that water under sufficiently high external pressure behaves like a normal liquid [3].

Most of the thermodynamic and kinetic anomalies of this liquid become more pronounced at low temperatures. The study of the liquid in the supercooled range, which extends from the melting pressure curve to the homogeneous nucleation pressure curve, thus contributes significantly to our understanding $[2-4]$ of the behaviour of the liquid in its stable phase. In addition, these studies are stringent tests for the various physical models applied to the description of the properties of supercooled water $[5,6]$.

Among the most pronounced anomalies of the dynamic properties of liquid water is the increase of translational mobility with initial compression of cold water, which is observed as an increase in the self diffusion coefficient and a decrease in viscosity. This behaviour is apparent already at temperatures above the equilibrium melting pressure curve. In this paper the self diffusion in supercooled light and heavy water has been studied with the NMR spin echo technique with pulsed field gradients [8], at pressures up to $400 \mathrm{MPa}$ and temperatures down to $203 \mathrm{~K}$. A previous

*) Permanent address: Department of Chemistry, Victoria University of Wellington, Wellington/New Zealand.

**) Please direct all correspondence concerning the manuscript to Prof. Dr. H.-D. Lüdemann at the address given above. paper reported the self diffusion coefficients of light water at pressures up to $300 \mathrm{MPa}$ and temperatures higher than $213 \mathrm{~K}$ [9]. The extension of the pressure and temperature range for light water and the inclusion of heavy water in the study given here allows a more detailed analysis of the data.

The study of the properties of supercooled water is most readily accomplished in emulsions of water in alkane mixtures [2]. In these emulsions the rotational correlation times of light and heavy water could be determined down to the homogeneous nucleation temperature curves [4]. The water droplets in these emulsions have diameters of approx. $1-10$ $\mu \mathrm{m}$.

The reliable measurement of the self diffusion coefficient demands, that the root mean square displacement of the molecules during the time needed for an experiment is small compared to the dimensions of the sample containing the liquid. This condition cannot be satisfied for the emulsions, as preliminary experiments showed. It was thus necessary to develop elaborate cleaning procedures and to use bulk water contained in thin capillaries for the determinations of $D$ given in this paper. A prerequisite for the study of selfdiffusion of water in the supercooled range is thus the reliable production of water of high purity.

\section{Experimental}

\section{Purification and Filling Procedure}

Light water was freshly drawn from a Milli-Q-unit equipped with prefilter-, carbon-, ion exchanger- and ultrafiltration-cartridges and a Millistak GS filter (Millipore, Eschborn, BRD). The water was degassed by several freeze pump thaw cycles and finally sublimed into a high vacuum filling apparatus. All parts of the apparatus, that came into contact with the liquid, were thoroughly rinsed with the high purity water in order to remove dust and other nucleating particles. The heavy water was drawn from freshly opened $10 \mathrm{ml}$ ampoules containing $99.95 \%$ deuterated water (Merck, Darmstadt, BRD). It was degassed on the high vacuum line and purified by at least two sublimations. The filling apparatus contains two to four high pressure glass capillaries of the design shown in Fig. 1. These are filled completely with the liquid, removed from the apparatus after flushing the vacuum part with argon, and flame sealed at the upper end.

\section{High Pressure Apparatus}

The capillaries are freshly drawn from Duran 50 glass and glued into the bore of the copper/beryllium nipple with an alumina filled epoxy resin. The teflon shrink hose serves as a pressure transmitter 


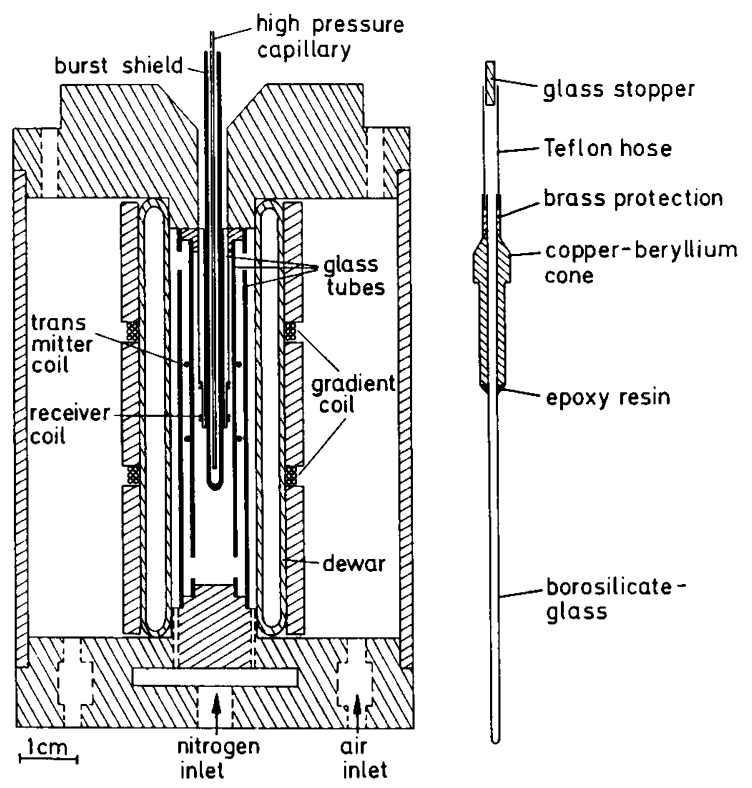

Fig. 1

Left: NMR probehead for measuring self diffusion coefficients of liquids at elevated pressures with a pulsed gradient. Right: High pressure cell drawn from Duran-50 glass capillary. O.D. of the glass: $800 \mu \mathrm{m}$; I.D. of the glass: $200 \mu \mathrm{m}$ or $80 \mu \mathrm{m}$

and separator. The nipple is screwed into a copper/beryllium autoclave and pressurized by commercial high pressure equipment. Details of the complete high pressure set-up have been published $[10-12]$.

The outer diameter of the capillaries was approx. $800 \mu \mathrm{m}$. The inner diameters varied between 200 and $70 \mu \mathrm{m}$. The tubes with the larger i.d. routinely withstood pressures up to $200 \mathrm{MPa}$, while a significant portion of the narrower tubes could be pressurized to $400 \mathrm{MPa}$. Because of the poor signal to noise ratio of the deuteron signal only the larger tubes could be used for the study of $\mathrm{D}_{2} \mathrm{O}$ thus limiting the pressure range for this substance to $200 \mathrm{MPa}$.

\section{Measurements}

The measurements were taken in a Bruker MSL 300 NMR spectrometer operating at a field of 7.05 Tesla. The home built probehead for the determination of the self diffusion coefficient with the pulsed field gradient spin echo technique proposed by Stejskal and Tanner [8] is shown in Fig. 1. The gradient is produced with a pair of opposite Helmholtz coils wound onto the outside wall of the insulating glass dewar. This design keeps the coil at room temperature and eliminates any temperature effect on the gradient strength. In order to avoid cooling or heating of the coil during prolonged temperature variations, room temperature air is blown through the annular space between coil and outer glass support. The tuning and matching of the probe was accomplished with nonmagnetic capacitors and coils arranged beneath the bottom plate of the probe. The pressure was measured with a Bourdon gauge (Heise, Connecticut, USA) to $\pm 0.5 \mathrm{MPa}$. The temperature was regulated with the Bruker variable temperature accessory and controlled in the space between the high pressure capillary and the burst shield at the level of the receiver coil before and after each measurement by a miniature thermocouple (Philips, Kassel, FRG).

In the presence of a pulsed field gradient, the decay of the echo amplitude $A$ in a Hahn-spin-echo experiment is given by [8]

$A(2 \tau)=A(0) \exp -\left(\frac{2 \tau}{T_{2}}\right) \exp \left(-\gamma^{2} D \delta^{2}\left(\Delta-\frac{1}{3} \delta\right) g^{2}\right)$

In Eq. (1) $\tau$ is the time between the $90^{\circ}$ and $180^{\circ}$ pulse, $\gamma$ the gyromagnetic ratio of the nucleus studied, $D$ the self diffusion coefficient, $\delta$ the duration of the gradient pulses, $\Delta$ the time between the two gradient pulses, and $g$ the gradient strength, given by $g=$ $k \cdot I$. Here $k$ means the coil constant, that has to be obtained from a calibration with a substance of known diffusion coefficient.

The determination of $D$ was most conveniently accomplished by increasing $g$ while keeping all other variables constant. The coil constant was determined using the known self diffusion coefficient of water at ambient pressure and $298 \mathrm{~K}$ obtained by Mills [13] which are generally acknowledged to be the most reliable values [14]. The coil constant was controlled with a redetermination of $D$ for benzene [15].

The self diffusion coefficients for $\mathrm{H}_{2} \mathrm{O}$ are judged reliable to $\pm 3 \%$. Their reproducability was $\pm 1-2 \%$ except for the temperatures below $210 \mathrm{~K}$ where the strong temperature dependence to. gether with the very short $T_{2}$ increases the maximal error to $\pm 10 \%$. In $\mathrm{D}_{2} \mathrm{O}$ the crror of the self diffusion coefficients, is because of the lower sensitivity of the deuterium nucleus for NMR experiments estimated to $\pm 5 \%$. Except for the lowest isotherm, where it increases to $\pm 10 \%$. The reproducibility was $\pm 2-3 \%$.

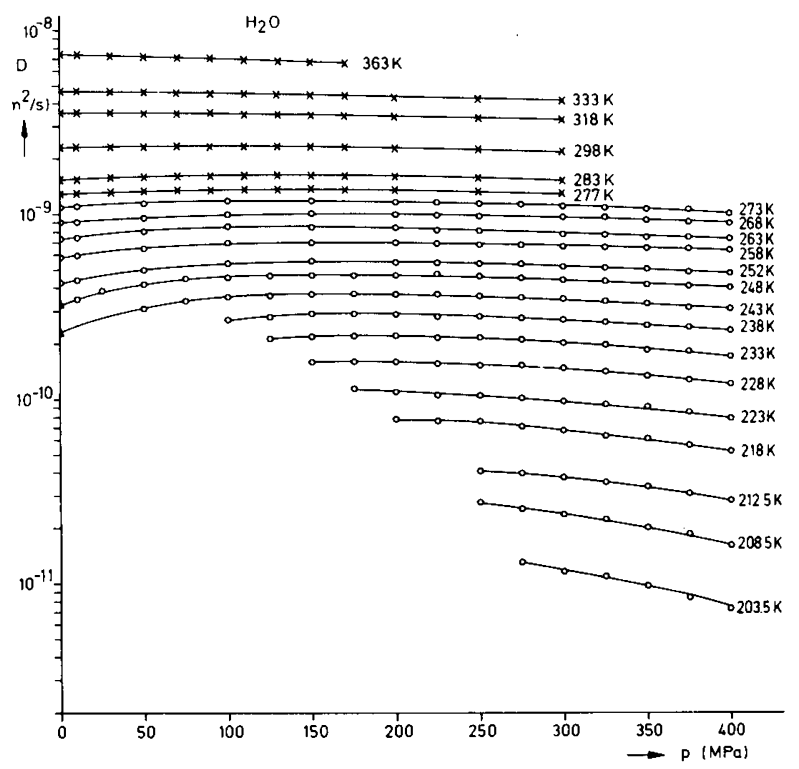

Fig. 2

Isotherms of the self diffusion coefficient of $\mathrm{H}_{2} \mathrm{O}$.

$\times$ Literature values $[14,15]$

$\bigcirc$ new measurements

$\triangle$ corrected data by Gillen et al. [16] for details see text

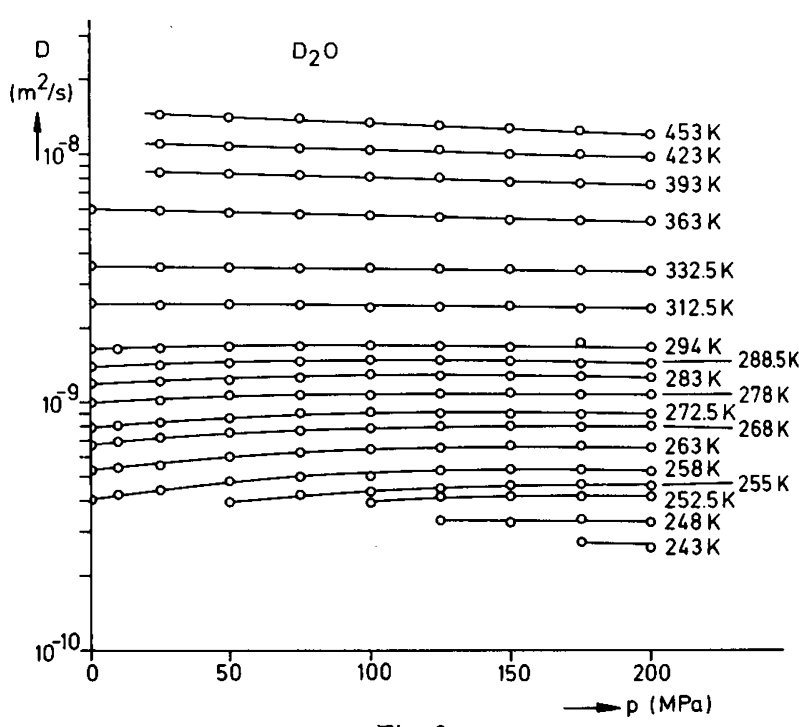

Fig. 3

Isotherms of the self diffusion coefficient of $\mathrm{D}_{2} \mathrm{O}$. The data obtained above the melting pressure curve agree with published results [17-19] 


\section{Results}

Fig. 2 compiles the self diffusion coefficients obtained for light water. Data for $T>273 \mathrm{~K}$ were taken from the Refs. $[16,17]$. At ambient pressure Gillen et al. [18] measured $D$ to temperatures as low as $242 \mathrm{~K}$. However, in the range of overlap with the data from other groups, between $277 \mathrm{~K}$ and $298 \mathrm{~K}$, their results are $\sim 7 \%$ lower than the accepted values compiled critically by Weingärtner [14]. The ambient pressure data points of the two lowest complete isotherms of Fig. 2 (open triangles) were thus taken from Gillens data and multiplied by a factor 1.07 .

Fig. 3 gives self diffusion data for deuterium oxide. Above the melting pressure curve our results agree with published data [19-21] within the limits of experimental error.

\section{Discussion}

\section{Pressure Dependence of Dynamic Properties}

In simple liquids application of pressure causes a decrease of the translational mobility. In water this normal behaviour is observed only at temperatures above $300 \mathrm{~K}$. In the low temperature range the measurements are limited to $p>200$ $\mathrm{MPa}$, since the proximity of the homogeneous nucleation pressure curve induces crystallisation on further lowering of the pressure. At these high pressures $D$ decreases with pressure.

In the temperature range $300 \mathrm{~K}>T>220 \mathrm{~K}$ water shows the well known anomaly, that mobility is enhanced by initial compression. At the lowest temperature where complete isotherms could be obtained ( $243 \mathrm{~K}$ for $\mathrm{H}_{2} \mathrm{O}$ ), $D$ increases between 0.1 and $150 \mathrm{MPa}$ by a factor of 1.6 .

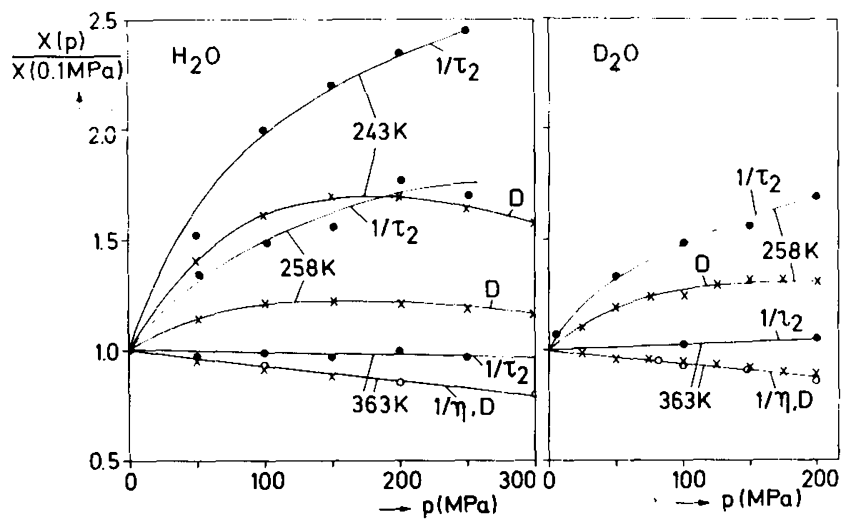

Fig. 4

Reduced isotherms for some transport properties of $\mathrm{H}_{2} \mathrm{O}$ (left) and $\mathrm{D}_{2} \mathrm{O}$ (right). The value of $X(p)$ at pressure $p$ relative to its atmospheric pressure value $(0.1 \mathrm{MPa})$ is plotted as function of pressure.

$\bigcirc$ filled circles $1 / \tau_{2}[21] ; \times$ crosses $D ; \bigcirc$ open circles reciprocal viscosity $1 / \eta$

Fig. 4 compares the pressure dependence of the self diffusion coefficient, characterizing translational motion, with the pressure dependence of $1 / \tau_{2}$, the inverse rotational correlation times, as obtained from oxygen-17-spin lattice relaxation time measurements by Lang and Lüdemann [21].

The $363 \mathrm{~K}$-isotherms reveal the pressure effects as observed for all normal liquids: Increasing the density reduces the translational mobility, as described by the viscosity $\eta$ or the self diffusion coefficient $D$, significantly stronger than the rotational diffusion characterized by $1 / \tau_{2}$. This normal behaviour is strikingly reversed in the supercooled region, where the translational mobility is enhanced along the 243 $\mathrm{K}$-isotherm of $\mathrm{H}_{2} \mathrm{O}$ by a factor of 1.6 while the inverse rotational correlation times increase by approximately a factor of 2.5. Also the pressure where the maximal mobility is observed along each isotherm appears to be significantly lower for $D(\sim 150 \mathrm{MPa})$ than for $1 / \tau_{2}(\sim 250 \mathrm{MPa})$.

All thermodynamic and dynamic anomalies of water originate from the open network structure of this liquid. The ability of the water molecule to participate with its two lone pairs and its two hydrogens in four hydrogen bonds enforces an approximately tetrahedral local arrangement with rather poor packing efficiency. Removal of thermal energy forces the molecules into low energy configurations with optimal relative orientations and distances which resemble local structures observed in low pressure polymorphs of ice and clathrates. The quasitetrahedral force field presented by the surrounding networks constrains a molecule to reside in one of a few well-defined orientational states which are separated by potential barriers. Because of reduced thermal excitations upon cooling the lifetime of these fully $\mathrm{H}$-bonded local structures must increase with a consequent slowing down of diffusive modes. Compression of the sample brings next nearest neighbours closer to a central molecule with nearest neighbour correlations being largely unchanged. The potential energy surface for rotation is then less sharply partitioned and there is a greater variety of orientational states, separated by smaller energy barriers, so that rotational diffusion is enhanced.

In all ice and clathrate networks each molecule is hydrogen bonded to four neighbours with bond length of $2.8 \pm$ $0.1 \AA[23,24]$. The significant difference is that whereas in the clathrates and in ice $I$, the distance of closest approach of the nonbonded neighbours is $\simeq 4.5 \AA$, in ices II, III, IV and $\mathrm{V}$ they lie in the range $3.2-3.5 \AA$. Studies of the ices show that dielectric relaxation, and hence rotational diffusion, is about 100 times faster in ices III, IV and V than in ice I. (lt is, of course, much slower in the proton ordered ice II). Because rotational correlation times are shorter than the corresponding translational correlation times $\left(\tau_{\mathrm{t}} \simeq\left\langle d^{2}\right\rangle /\right.$ $6 D, d \simeq 3 \AA$ ), orientational fluctuations initiate structural rearrangements within the transient $\mathrm{H}$-bond network. Consequently, the enhancement of local molecular reorientations upon initial compression must facilitate also translational motions. However, an increasing number of next neighbours must form obstacles for translational motions. Thus self-diffusion starts to decrease already at pressures where rotation is still enhanced by further compression.

\section{Temperature Dependence of Self-Diffusion}

Like most dynamic properties of water, the isobars of the self diffusion coefficient also reveal a pronounced nonlinearity in Arrhenius plots, with the slope increasing strongly with falling temperature. This strong temperature dependence of a dynamic response to small perturbations from equilibrium is generally observed in dense, undercooled liquids. It can often be accommodated either to a dynamic scaling law [25]

$X(T) \sim\left(T-T_{0}\right)^{-\gamma}$ 
or to a modified Arrhenius (VTF) equation [26-28]

$X(T) \sim \exp \left(-\frac{B}{T-T_{0}}\right)$

The respective temperatures $T_{0}$ are interpreted to signify a kinetic localisation phenomenon on the time-scales of relevance.

Because of the apparently diverging correlation length of structural fluctuations in supercooled water at low pressures Speedy and Angell [29] suggested a dynamic scaling behaviour of the form

$D(T)=D_{0}\left(T / T_{\mathrm{s}}-1\right)^{\gamma}$.

Here $T_{\mathrm{s}}$ represents a low temperature limit of the fluid phase, where $D$ extrapolates to zero. This power law description has been applied successfully to many thermodynamic and kinetic properties of supercooled water $[2,3]$. The singular temperatures $T_{\mathrm{s}}$ turned out to fall approx. $10 \mathrm{~K}$ below the homogeneous nucleation temperature $T_{\mathrm{H}}(p=0.1 \mathrm{MPa})$ in all cases. Accordingly $T_{\mathrm{s}}(p)$ has been interpreted by Speedy [5] as a spinodal line or locus of the mechanical stability limit.

In principle a factor of $T^{1 / 2}$ should be included in Eq. (2) because the diffusion coefficient varies in proportion to the speed of the molecules. Thus, if the density and structure were not influenced by $T$ then $D T^{-1 / 2}$ would be constant as is the case in the hard sphere fluid for example, and it is the influence of variations in the structure on $D T^{-1 / 2}$ that one seeks to describe by the power law or VTF term. The $T^{1 / 2}$ term was included in Ref. [9], but in practice it makes a relatively small contribution to the total temperature derivative of $D$ and it can be absorbed into the more strongly varying terms with little effect on the parameter values.

In many dense, highly viscous liquids with tendency for supercooling the VTF-equation [26-28] can be applied to represent the slowing down of structural fluctuations according to

$D(T)=D_{0} \exp -\left(\frac{B}{T-T_{0}}\right)$.

Here $T_{0}$ means the ideal glass transition temperature, where diffusion ceases to occur. To be consistent, $T_{0}$ must be related to the Kauzmann temperature [31], where the configurational entropy of the amorphous and crystalline phases would match.

Tables 3 and 4 give the parameters of the VTF-equation as obtained from a least squares fit of Eq. (3) to the selfdiffusion data of Tables 1 and 2 . The corresponding parameters for the fractional power law description are compiled in Tables 5 and 6.

Comparison of the standard deviations found for each fit does not permit a decision in favour of one of the two descriptions proposed. However, not all best fit parameters seem to be acceptable physically.

For the low pressure isobars, the $T_{0}$ obtained from the VTF-equation are far above any observed or extrapolated
Table 1

Self diffusion coefficient $D\left(10^{-10} \mathrm{~m}^{2} \cdot \mathrm{s}^{-1}\right)$ in $\mathrm{H}_{2} \mathrm{O}$

\begin{tabular}{cccccccccc}
\hline$p(\mathrm{MPa})$ & 0.1 & 50 & 100 & 150 & 200 & 250 & 300 & 350 & 400 \\
\hline$T(\mathrm{~K})$ & & & & & & & & & \\
\hline 273.0 & 10.9 & 11.6 & 11.7 & 11.8 & 11.8 & 11.6 & 10.9 & 10.7 & 10.2 \\
268.0 & 9.00 & 9.73 & 10.0 & 10.2 & 9.90 & 9.80 & 9.65 & 9.27 & 8.88 \\
263.0 & 7.42 & 8.08 & 8.55 & 8.51 & 8.47 & 8.15 & 7.78 & 7.52 & 7.33 \\
258.0 & 5.75 & 6.55 & 6.94 & 7.02 & 6.97 & 6.77 & 6.67 & 6.59 & 6.38 \\
255.0 & 4.94 & 5.71 & 6.15 & 6.30 & 6.22 & 6.10 & 5.82 & 5.63 & 5.38 \\
252.0 & 4.29 & 5.04 & 5.42 & 5.55 & 5.51 & 5.38 & 5.25 & 5.02 & 4.76 \\
248.0 & & 4.15 & 4.51 & 4.65 & 4.72 & 4.58 & 4.39 & 4.18 & 4.05 \\
243.0 & & 3.11 & 3.56 & 3.70 & 3.70 & 3.60 & 3.47 & 3.27 & 3.08 \\
238.0 & & & 2.70 & 2.90 & 2.90 & 2.80 & 2.66 & 2.48 & 2.34 \\
233.0 & & & & 2.18 & 2.26 & 2.18 & 2.02 & 1.87 & 1.72 \\
228.0 & & & & 1.61 & 1.58 & 1.52 & 1.42 & 1.34 & 1.21 \\
223.0 & & & & & 1.08 & 1.05 & 0.980 & 0.900 & 0.785 \\
218.0 & & & & & 0.762 & 0.710 & 0.670 & 0.607 & 0.519 \\
212.0 & & & & & & 0.412 & 0.380 & 0.335 & 0.287 \\
208.5 & & & & & & 0.276 & 0.236 & 0.197 & 0.158 \\
203.5 & & & & & & & 0.118 & 0.099 & 0.075 \\
& & & & & & & & &
\end{tabular}

Table 2

Self diffusion coefficient $D\left(10^{-10} \mathrm{~m}^{2} \cdot \mathrm{s}^{-1}\right)$ in $\mathrm{D}_{2} \mathrm{O}$

\begin{tabular}{|c|c|c|c|c|c|c|c|c|c|}
\hline$\frac{p(\mathrm{MPa})}{T(\mathrm{~K})}$ & 0.1 & 25 & 50 & 75 & 100 & 125 & 150 & 175 & 200 \\
\hline 453.0 & & 144 & 143 & 140 & 136 & 131 & 128 & 125 & 122 \\
\hline 423.0 & & 110 & 109 & 107 & 106 & 104 & 101 & 101 & 97.6 \\
\hline 393.0 & & 85.3 & 84.2 & 82.6 & 82.3 & 79.8 & 78.0 & 76.9 & 75.8 \\
\hline 363.0 & 60.5 & 59.4 & 58.3 & 58.0 & 57.1 & 56.1 & 55.4 & 54.6 & 53.8 \\
\hline 332.5 & 35.7 & 35.0 & 35.3 & 34.9 & 34.9 & 34.6 & 34.5 & 34.4 & 34.1 \\
\hline 312.5 & 24.9 & 24.8 & 25.1 & 24.8 & 24.1 & 24.4 & 24.5 & 24.1 & 23.8 \\
\hline 294.0 & 16.6 & 16.8 & 17.0 & 17.0 & 17.1 & 17.0 & 16.7 & 16.5 & 16.7 \\
\hline 288.5 & 14.0 & 14.2 & 14.5 & 14.8 & 15.0 & 15.9 & 14.8 & 14.6 & 14.5 \\
\hline 283.0 & 12.1 & 12.3 & 12.4 & 12.6 & 13.0 & 12.7 & 12.7 & 12.7 & 12.6 \\
\hline 278.0 & 10.0 & 10.3 & 10.7 & 10.7 & 10.7 & 10.9 & 10.9 & 10.7 & 10.7 \\
\hline 272.5 & 7.89 & 8.28 & 8.76 & 9.14 & 9.15 & 9.07 & 9.05 & 9.00 & 8.90 \\
\hline 268.0 & 6.73 & 7.28 & 7.58 & 7.76 & 7.88 & 7.98 & 8.02 & 8.01 & 8.00 \\
\hline 263.0 & 5.35 & 5.61 & 6.08 & 6.25 & 6.49 & 6.53 & 6.71 & 6.73 & 6.61 \\
\hline 258.0 & 4.06 & 4.46 & 4.84 & 5.02 & 5.05 & 5.30 & 5.36 & 5.35 & 5.33 \\
\hline 255.0 & & & 3.96 & 4.25 & 4.40 & 4.52 & 4.63 & 4.71 & 4.60 \\
\hline 252.5 & & & & & 3.94 & 4.18 & 4.19 & 4.21 & 4.18 \\
\hline 248.0 & & & & & & 3.36 & 3.29 & 3.42 & 3.28 \\
\hline 243.0 & & & & & & & & 2.76 & 2.60 \\
\hline
\end{tabular}

glass transition temperature of $\mathrm{H}_{2} \mathrm{O}$ and $\mathrm{D}_{2} \mathrm{O}\left(T_{\mathrm{g}}<140 \mathrm{~K}\right)$ [32]. Calorimetric glass transition temperatures $T_{\mathrm{g}}$ are higher than $T_{0}$ due to finite cooling rates. Using small sample techniques, water could indeed be vitrified and displayed a weak glass transition at $136 \mathrm{~K}$ [33]. Extrapolations to zero concentration of calorimetric glass transitions $T_{\mathrm{g}}(c)$, observed in aqueous solutions, suggest $\lim _{c \rightarrow 0} T_{\mathrm{g}}(c) \simeq 139 \mathrm{~K}$ in $\mathrm{H}_{2} \mathrm{O}$ and $144 \mathrm{~K}$ in $\mathrm{D}_{2} \mathrm{O}[34,35]$ at ambient pressure. For water at $p=200 \mathrm{MPa}$ a transition temperature $T_{\mathrm{g}}=146 \mathrm{~K}$ has been estimated by extrapolating corresponding $T_{\mathrm{g}}$ data of $\mathrm{NaCl}+\mathrm{H}_{2} \mathrm{O}$ and $\mathrm{LiCl}+\mathrm{H}_{2} \mathrm{O}$ glasses. Also $T_{0}=130 \mathrm{~K}$ has been deduced for $\mathrm{D}_{2} \mathrm{O}$ at $p=200 \mathrm{MPa}$ from $C_{p}$ measurements under pressure [36].

At high pressures ( $p>150 \mathrm{MPa}$ ), on the other hand, the power law description leads to singularity temperatures $T_{\mathrm{s}}$ that are higher than the respective homogeneous nucleation 
Table 3

Best fit parameters for the description of the isobaric temperature dependence of $D$ in $\mathrm{H}_{2} \mathrm{O}$ according to $D=D_{0} \exp \left(-B / T-T_{0}\right)$. In the $p, T$ region above the melting pressure curve, the accepted published self diffusion coefficients [13] were used for the fits. $T_{0}$ values in parentheses were derived from the $\tau_{2}$-data $[22]$

\begin{tabular}{cccclc}
$P[\mathrm{MPa}]$ & $T[\mathrm{~K}]$ & $\begin{array}{c}D_{0} \cdot 10^{8} \\
{\left[\mathrm{~m}^{2} / \mathrm{s}\right]}\end{array}$ & $B\left[\mathrm{~K}^{-1}\right]$ & $T_{0}[\mathrm{~K}]$ & Std. dev.\% \\
\hline 0.1 & $242-333$ & 4.14 & 347 & 177 & 3.1 \\
10 & $252-333$ & 6.46 & 455 & 161 & 1.3 \\
50 & $243-333$ & 8.90 & 563 & 143 & 0.6 \\
100 & $238-333$ & 10.1 & 622 & 133 & 0.7 \\
150 & $228-333$ & 11.2 & 668 & $126(131)$ & 1.0 \\
200 & $218-333$ & 8.93 & 614 & $131(134)$ & 1.5 \\
250 & $208.5-333$ & 7.24 & 564 & $137(135)$ & 2.6 \\
300 & $203.5-333$ & 5.78 & 514 & 142.5 & 3.6 \\
350 & $203.5-273$ & 3.41 & 423 & 152 & 2.6 \\
400 & $203.5-273$ & 3.24 & 410 & 154.5 & 2.1 \\
\hline
\end{tabular}

Table 4

Best fit parameters for the description of the isobaric temperature dependence of $D$ in $\mathrm{D}_{2} \mathrm{O}$ according to $D=D_{0} \exp \left(-B / T-T_{0}\right)$. In the $p . T$ region above the melting pressure curve, the accepted published self diffusion coefficients [13] were used for the fits. $T_{0}$ values in parentheses were derived from the $\tau_{2}$-data $[22]$

\begin{tabular}{cccccc}
\hline$P[\mathrm{MPa}]$ & $T[\mathrm{~K}]$ & $\begin{array}{c}D_{0} \cdot 10^{\mathrm{x}} \\
{\left[\mathrm{m}^{2} / \mathrm{s}\right]}\end{array}$ & $B\left[\mathrm{~K}^{-1}\right]$ & $T_{0}[\mathrm{~K}]$ & Std. dev.\% \\
\hline 0.1 & $258-363$ & 6.63 & 493 & $161(185)$ & 2.8 \\
25 & $258-453$ & 12.1 & 687 & 135 & 3.3 \\
50 & $255-453$ & 11.3 & 671 & $135(165)$ & 3.5 \\
75 & $255-453$ & 11.7 & 700 & 129 & 3.2 \\
100 & $252.5-453$ & 10.7 & 678 & $131(140)$ & 3.5 \\
125 & $248-453$ & 10.9 & 697 & 127 & 2.5 \\
150 & $248-453$ & 9.93 & 671 & $130(133)$ & 2.4 \\
175 & $243-453$ & 10.6 & 706 & 125 & 2.0 \\
200 & $243-453$ & 8.92 & 645 & $132(139)$ & 2.3 \\
\hline
\end{tabular}

Table 5

Best fit parameters for the description of the isobaric temperature dependence of $D$ in $\mathrm{H}_{2} \mathrm{O}$ according to $D=D_{0}\left(T / T_{\mathrm{s}}-1\right)^{\prime}$. In the $p, T$ region above the melting pressure curve. the accepted published self diffusion coefficients [13] were used for the fits. $T_{\mathrm{s}}$ values in parentheses were derived from the $\tau_{2}$-data [22]

\begin{tabular}{ccclcc}
$P[\mathrm{MPa}]$ & $T[\mathrm{~K}]$ & $\begin{array}{l}D_{0} \cdot 10^{8} \\
{\left[\mathrm{~m}^{2} / \mathrm{s}\right]}\end{array}$ & \multicolumn{1}{c}{$T_{\mathrm{s}}[\mathrm{K}]$} & $\gamma$ & Std. dev.\% \\
\hline 0.1 & $242-333$ & 1.67 & $223(223)$ & 1.82 & 2.4 \\
10 & $252-333$ & 1.61 & 219 & 1.92 & 0.8 \\
50 & $243-333$ & 1.37 & $208(215)$ & 2.12 & 0.8 \\
100 & $238-333$ & 1.20 & $203(207)$ & 2.19 & 1.0 \\
150 & $228-333$ & 0.99 & $193(190)$ & 2.42 & 1.8 \\
200 & $218-333$ & 0.93 & 191 & 2.48 & 1.8 \\
250 & $208.5-333$ & 0.90 & 189.5 & 2.54 & 2.5 \\
300 & $203.5-333$ & 0.90 & 189 & 2.60 & 2.7 \\
350 & $203.5-273$ & 0.99 & 188 & 2.78 & 2.7 \\
400 & $203.5-273$ & 1.06 & 189 & 2.82 & 2.8 \\
\hline
\end{tabular}

temperatures $T_{\mathrm{H}}$. This result is obviously in contradiction to the interpretation of $T_{\mathrm{s}}$ as the temperature of the limit of mechanical stability. Attempts to use $T_{\mathrm{s}}$-values approx. $10 \mathrm{~K}$ lower than $T_{\mathrm{H}}$ lead to a poor fit for the diffusion coefficients at the highest temperatures $(T>300 \mathrm{~K})$. This might not be
Table 6

Best fit parameters for the description of the isobaric temperature dependence of $D$ in $\mathrm{D}_{2} \mathrm{O}$ according to $D=D_{0}\left(T / T_{\mathrm{s}}-1\right)^{\gamma}$. In the $p, T$ region above the melting pressure curve, the accepted published self diffusion coefficients [13] were used for the fits. $T_{\mathrm{s}}$ values in parentheses were derived from the $\tau_{2}$-data [22]

\begin{tabular}{clllll}
$P[\mathrm{MPa}]$ & $T[\mathrm{~K}]$ & $\begin{array}{c}D_{0} \cdot 10^{8} \\
{\left[\mathrm{~m}^{2} / \mathrm{s}\right]}\end{array}$ & $T_{\mathrm{s}}[\mathrm{K}]$ & $\gamma$ & Std. dev.\% \\
\hline 0.1 & $258-363$ & 1.48 & $226(230)$ & 1.83 & 2.1 \\
25 & $258-453$ & 1.37 & 223 & 1.85 & 2.2 \\
50 & $255-453$ & 1.31 & $221(221)$ & 1.85 & 1.9 \\
75 & $255-453$ & 1.23 & 219 & 1.86 & 1.9 \\
100 & $252.5-453$ & 1.19 & $218(207)$ & 1.85 & 2.4 \\
125 & $248-453$ & 1.09 & 214 & 1.90 & 1.9 \\
150 & $248-453$ & 1.08 & $215(197)$ & 1.86 & 1.5 \\
175 & $243-453$ & 0.99 & 210 & 1.95 & 2.3 \\
200 & $243-453$ & 1.02 & $214(188)$ & 1.87 & 2.2 \\
\hline
\end{tabular}

surprising as the power law is expected to be most appropriate close to the transition. Rather it is astonishing that the data at low pressure display a power law dependence over so large a temperature range (see Table 5). This is also true for other dynamic properties of cold water [2-4].

Contrary to $T_{\mathrm{s}}$ the $T_{0}$ derived at pressures $p>150 \mathrm{MPa}$ from the fit to the VTF-equation are within the range of estimated glass transition temperatures. The important difference to the power law description being that the data conform to the VTF-law over the whole temperature range investigated.

Both data sets presented here, however, end for technical reasons, well above the homogeneous nucleation temperature. But the fitting to either of the two Eqs. (2) and (3) is most sensitive to results at the lowest temperatures obtainable. Hence some of the above mentioned inconsistencies are probably an artefact of the fitting exercise due to a lack of data sufficiently close to $T_{\mathrm{H}}$ (hence $T_{\mathrm{s}}$ ). The most stringent tests for the dynamic properties, that could be obtained hitherto, were the determination of the rotational correlation times $\tau_{2}$ of the deuterons in heavy water and the oxygen-17 in light and heavy water. These measurements could be extended to within $\sim 1 \mathrm{~K}$ of the homogeneous nucleation pressure curve [22]. The $T_{0}$ resp. $T_{\mathrm{s}}$ values obtained from the fitting of the VTF-equation and the power-law to these data are therefore considered more reliable. The characteristic temperatures obtained from these treatments are given in parentheses in the Tables 3 to 6 . For pressures $p \leq 150$ $\mathrm{MPa}$ the $T_{\mathrm{s}}$-values agree with the values obtained in this work, whereas the differences for $\mathrm{D}_{2} \mathrm{O}$ are much larger, due to the longer extrapolations involved in fitting the diffusion coefficients.

Fig. 5 compiles the singular temperatures $T_{s}\left(\mathrm{H}_{2} \mathrm{O}\right)$ in a supplemented phase diagram. The conclusion from the discussion concerning $T_{\mathrm{s}}$ and $T_{0}$ may be that water at pressures higher than $\sim 250 \mathrm{MPa}$ [32] behaves similar to a normal supercooled liquid. It is noteworthy in this respect, that the stability limit conjecture for $T_{\mathrm{s}}$ implies a diverging correlation length of structural fluctuations. On the other hand an important aspect of the glass transition, that distinguishes it from the dynamical "slowing down" near a critical point is that equilibrium correlation functions remain spatially 


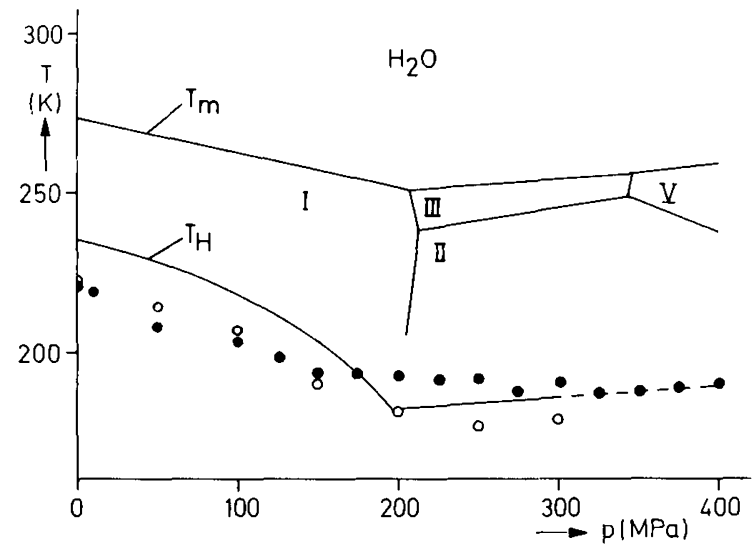

Fig. 5

Part of the phase diagram of $\mathrm{H}, \mathrm{O}$. The roman numbers indicate the stability regions of the various ice phases. $T_{\mathrm{m}}=$ Melting pressure curve; $T_{\mathrm{H}}=$ Homogeneous nucleation pressure curve; full circles: $T_{\mathrm{s}}$ as obtained from self diffusion coefficients; open circles:

$T_{\mathrm{s}}$ as obtained from rotational correlation times $\tau_{2}[20]$

short ranged near $T_{0}$ [37]. Hence one might consider hydrostatic pressure as a network breaking agent which suppresses long-range structural correlations. In this context recent hydrodynamic theories of the glass-transition [37-42] should be mentioned. They obtain power-law divergences of transport-coefficients near the freezing transition $T_{0}$. The power-law exponents $\gamma$ estimated from these theories come close to those obtained by fitting low pressure transport and relaxation coefficients of supercooled water to Eq. (2). This raises the question whether these modecoupling theories, applied to supercooled water at low pressure, could yield a dynamic scaling behaviour of transport and relaxation coefficients (see Eq. (2)) with $T_{\mathrm{s}}$ corresponding to a spinodal rather than a glass-transition as experimental evidence suggests. In the high pressure regime, however, the best-fit transition temperatures $T_{\mathrm{s}}$ turn out to lie above the respective nucleation temperatures $T_{\mathrm{H}}$ and cannot, therefore, be interpreted as spinodals any further. But the transition temperatures $T_{\mathrm{s}}$ also lie above any glass-transition temperatures $T_{0}$ deduced from application of Eq. (3) to the data. These observations are closely related to recent findings [43] that transport coefficients in many molecular liquids display power-law temperature dependences with $\gamma$ close to 2 at high temperatures as predicted by hydrodynamic theories but with transition temperatures well above any experimentally determined glass transitions. Thus the physical nature of the transition temperature in these liquids has yet to be clarified.

\section{Dynamic Isotope Effect}

The dynamic isotope effect for self diffusion defined by $D\left(\mathrm{H}_{2} \mathrm{O}\right) / D\left(\mathrm{D}_{2} \mathrm{O}\right)$ is given for three isobars in Fig. 6a. This ratio shows a significant increase with falling temperature. Within the limits of error the ratio of $1 / \tau_{2}$ for light and heavy water is identical to the ratio of the self diffusion coefficients [22], although one would expect the first to scale, at least in the range where the $T$-dependence is very weak, with the square root of the moments of inertia $(=1.38)$ and the second to depend on the square root of the mass ratios $(=1.05)$. Obviously the temperature- and pressure-dependence of self-diffusion in water is dominated by cooperative properties like hydrogen bond strength or local structures and not by single particle properties.

From the analysis of the relaxation data followed, that the difference between the correlation times $\tau_{2}$ for constant $T$ and $p$ in $\mathrm{H}_{2} \mathrm{O}$ and $\mathrm{D}_{2} \mathrm{O}$ originates only from a shift in the temperature $T_{\mathrm{s}}$ for the two substances, $T_{\mathrm{s}}\left(\mathrm{D}_{2} \mathrm{O}\right)$ being at the respective pressures $7 \mathrm{~K}$ higher than $T_{5}\left(\mathrm{H}_{2} \mathrm{O}\right)$ (Fig. 6b). Fig. 7 shows the self diffusion data for $\mathrm{H}_{2} \mathrm{O}$ and $\mathrm{D}_{2} \mathrm{O}$ in the power law description $\log D$ vers. $\log \left(T / T_{\mathrm{s}}-1\right)$. For $\mathrm{H}_{2} \mathrm{O}$ the best fit parameters for $T_{\mathrm{s}}$ were taken from Table 5 , the $T_{\mathrm{s}}$-values of $\mathrm{D}_{2} \mathrm{O}$ were assumed to be $7 \mathrm{~K}$ higher than the corresponding values in $\mathrm{H}_{2} \mathrm{O}$. In this representation all iso-

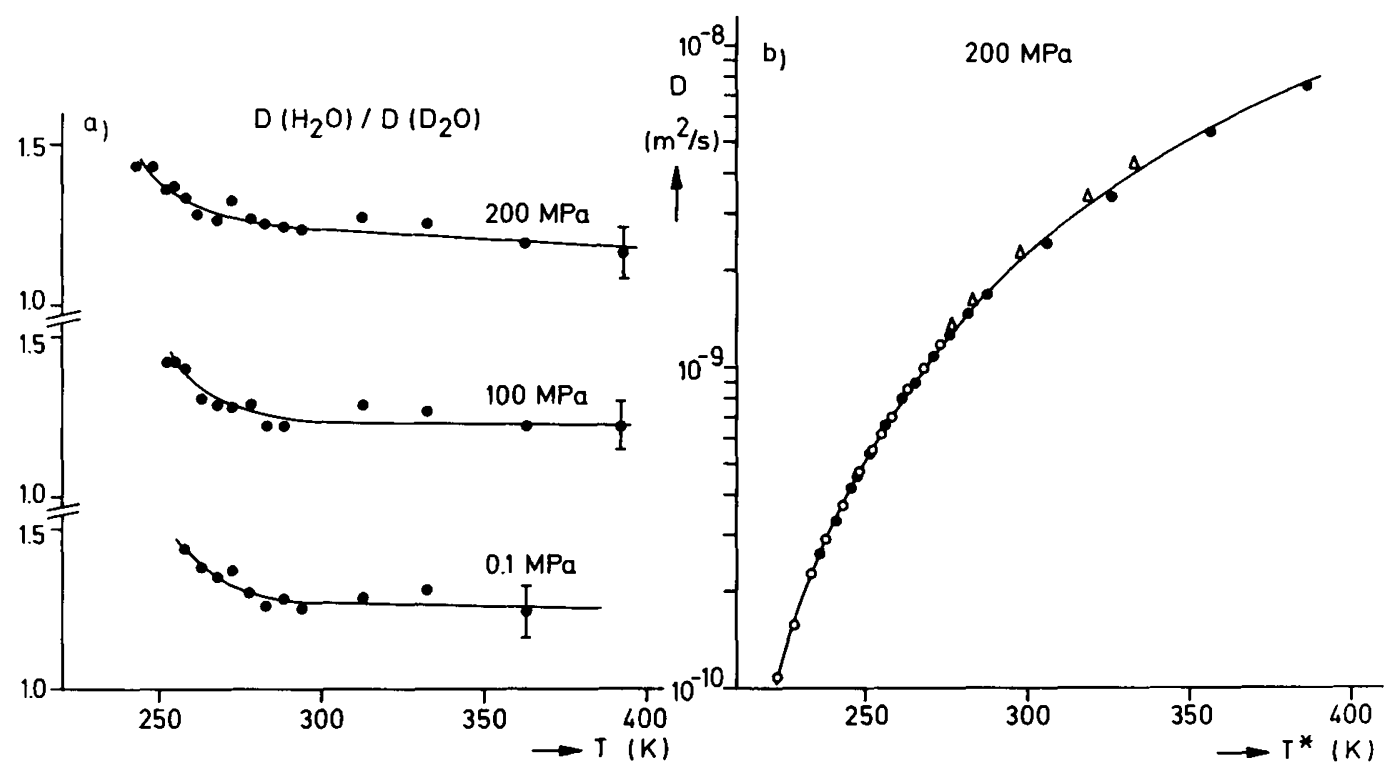

Fig. 6

a) Dynamic isotope effect described by the ratio of the self diffusion coefficients $D$ of $\mathrm{H}_{2} \mathrm{O}$ and $\mathrm{D}_{2} \mathrm{O}$ at three pressures.

b) Diffusion coefficient $D$ versus $T$ at $p=200 \mathrm{MPa}$ for $\mathrm{H}_{2} \mathrm{O}\left(T \equiv T^{*}\right)$ and $\mathrm{D}_{2} \mathrm{O}\left(T^{*}=T+7 \mathrm{~K}\right)$. 


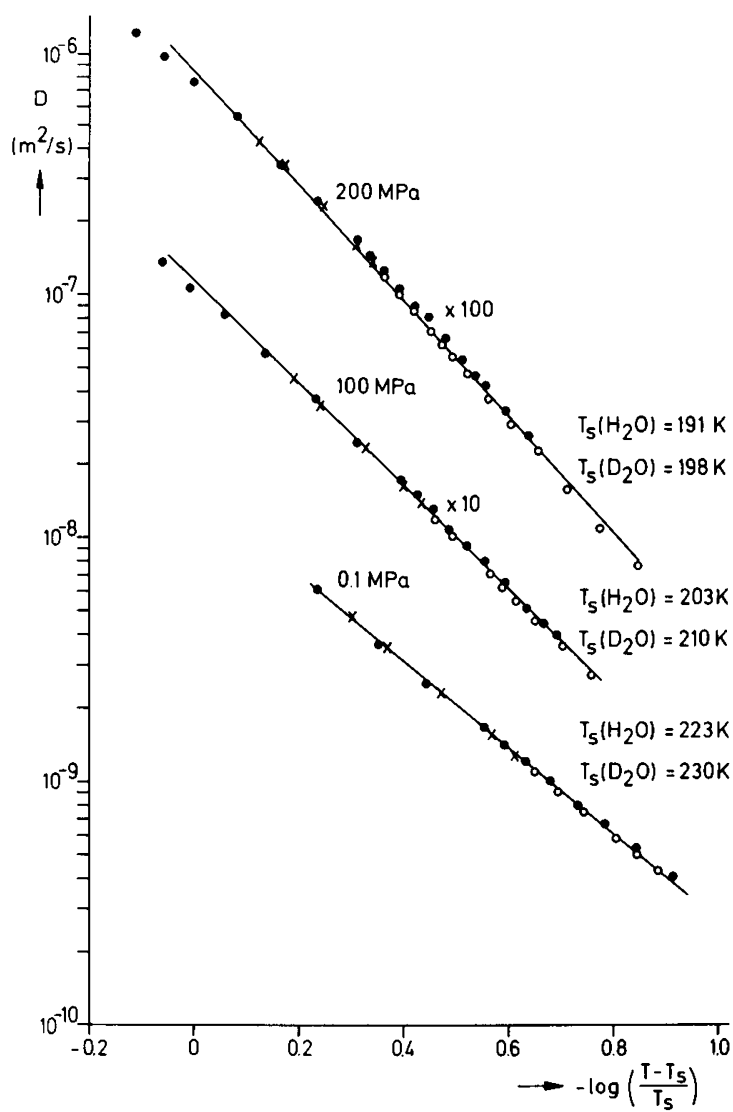

Fig. 7

Representation of the self diffusion coefficients $D$ by Eq. (2). The respective singularity temperatures are given in the figure

tope effects are scaled out. The difference of $7 \mathrm{~K}$ is also found for the homogeneous nucleation temperatures $T_{\mathrm{H}}$ in $\mathrm{H}_{2} \mathrm{O}$ and $\mathrm{D}_{2} \mathrm{O}$. Also the melting temperature $T_{\mathrm{m}}$ and the temperature of maximum density TMD are higher in $\mathrm{D}_{2} \mathrm{O}$, as well as extrapolated glass transition temperatures $T_{\mathrm{g}}$. The higher temperatures in $\mathrm{D}_{2} \mathrm{O}$ result from smaller amplitudes of zero-point vibrations, which cause stronger hydrogen bonds in heavy water. Shifting all temperature scales by $\sim 7 \mathrm{~K}$ in going from $\mathrm{H}_{2} \mathrm{O}$ to $\mathrm{D}_{2} \mathrm{O}$ removes most of the isotope effects observed (Fig. 6b).

Excellent technical support by S. Heyn, R. Knott, G. Niesner and G. Wührl made this work possible. Financial support by the DFG and the Fonds der Chemischen Industrie is gratefully acknowledged. F. X. Prielmeier thanks the Friedrich-Ebert-Stiftung for a scholarship.

\section{References}

[1] F. Franks, in: Water - A Comprehensive Treatise, Vol. 1, p. 1, ed. F. Franks, Plenum Press, New York 1972.

[2] C. A. Angell, in: Water - A Comprehensive Treatise, Vol. 7, p. 1, ed. F. Franks, Plenum Press, New York 1983.

[3] C. A. Angell, Ann. Rev. Phys. Chem. 34, 593 (1983).
[4] E. W. Lang and H.-D. Lüdemann, Angew. Chem. 94, 351 (1982); Angew Chem. I. Ed. 21, 315 (1982).

[5] R. J. Speedy, J. Phys. Chem. 86, 982 (1982).

[6] H. E. Stanley and J. Teixeira, J. Chem. Phys. 73, 3404 (1980).

[7] L. Bosio, J. Teixeira, and H. E. Stanley, Phys. Rev. Lett. 46, 597 (1981)

[8] E. O. Stejskal and J. E. Tanner, J. Chem. Phys. 42, 288 (1965).

[9] F. X. Prielmeier, E. W. Lang, R. J. Speedy, and H.-D. Lüdemann, Phys. Rev. Lett. 59, 1128 (1987).

[10] H. Yamada, Rev. Sci. Instrum. 45, 690 (1974).

[11] G. Völkel, E. W. Lang, and H.-D. Lüdemann, Ber. Bunsenges. Phys. Chem. 83, 722 (1979).

[12] U. Gaarz and H.-D. Lüdemann, Ber. Bunsenges. Phys. Chem. 80, 607 (1976).

[13] R. Mills, J. Phys. Chem. 77, 685 (1973).

[14] M. Weingärtner, Z. Phys. Chem. Neue Folge 132, 129 (1982).

[15] H. J. V. Tyrrell and K. R. Harris, Diffusion in Liquids, p. 227, Butterworths, London 1984.

[16] K. Krinicki, C. D. Green, and W. D. Sawyer, Faraday Discuss. Chem. Soc. 66, 199 (1980).

[17] K. R. Harris and L. A. Woolf, J. Chem. Soc. Faraday Trans. 1, 76, 377 (1980).

[18] K. T. Gillen, D. C. Douglass, and M. J. R. Hoch, J. Chem. Phys. 57, 5117 (1972).

[19] L. A. Woolf, J. Chem. Soc. Faraday Trans. 1, 1267 (1976).

[20] D. J. Wilbur, T. de Fries, and J. Jonas, J. Chem. Phys. 65, 1783 (1976).

[21] C. A. Angell, E. D. Finch, L. A. Woolf, and P. Bach, J. Chem. Phys. 65, 3063 (1976).

[22] E. W. Lang and H.-D. Lüdemann, Ber. Bunsenges. Phys. Chem. 85, 603 (1981)

[23] R. K. Chen, D. W. Davidson, and E. Whalley, J. Chem. Phys. 43, $2376(1965)$

[24] G. J. Wilson, R. K. Chen, D. W. Davidson, and E. Whalley, J. Chem. Phys. 43, 2384 (1965).

[25] R. G. Palmer, in: "Heidelberg Colloquium on Glassy Dynamics", Lecture Notes in Physics, Vol. 275, eds. J. L. van Hemmen and I. Morgenstern, Springer, Berlin 1987.

[26] H. Vogel, Phys. Z. 22, 645 (1921).

[27] G. Tammann and W. Hesse, Z. Anorg. Chem. 156, 245 (1926).

[28] G. S. Fulcher, J. Am. Ceram. Soc. 77, 3701 (1925).

[29] R. J. Speedy and C. A. Angell, J. Chem. Phys. 65, 851 (1976).

[30] C. T. Moynihan, D. L. Garin, and R. Syed, J. Phys. (Paris) C9 43, 395 (1982).

[31] W. Kauzmann, Chem. Revs. 43, 2191 (1948).

[32] C. A. Angell, Nature 331, 206 (1988).

[33] G. P. Johari, A. Hallbrucker, and E. Mayer, Nature 330, 552 (1987).

[34] C. A. Angell and E. J. Sare, J. Chem. Phys. 52, 1058 (1970).

[35] H. Kanno, I. Shirotani, and S. Minomura, Bull. Chem. Soc. Jpn. 53, 2079 (1980).

[36] H. Kanno and C. A. Angell, J. Chem. Phys. 73, 1940 (1980).

[37] G. H. Fredrickson and H. C. Andersen, Phys. Rev. Lett. 53, 1244 (1984).

[38] E. Leutheusser, Phys. Rev. A29, 2765 (1984).

[39] U. Bengtzelius, W. Götze, and A. Sjölander, J. Phys. C17, 5915 (1982)

[40] P. Papon and P. H. E. Meijer, Physica $101 \mathrm{~A}, 477$ (1980).

[41] S. P. Das, G. F. Mazenko, S. Ramaswamy, and J. J. Toner, Phys. Rev. Lett. 54, 118 (1985).

[42] T. R. Kirkpatrick, Phys. Rev. A31, 939 (1985).

[43] P. Taborek, R. N. Kleiman, and D. J. Bishop, Phys. Rev. B34, 1835 (1986).

(Eingegangen am 21. April 1988)

E 6785 